Military Technical College Kobry El-Kobbah, Cairo, Egypt

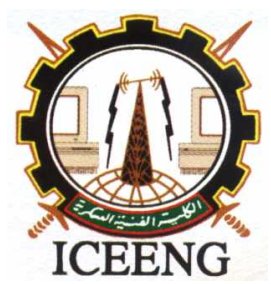

\author{
$6^{\text {th }}$ International Conference \\ on Electrical Engineering \\ ICEENG 2008
}

\title{
A computer interface for the assessment of the effect of reaction time on hand deviation (Tremor)
}

\author{
By \\ Mustafa Muheilan *
}

\section{Abstract:}

Subjects were asked to draw a line of fixed length, through a prescribed horizontal track using the light pen which is a computer interface (similar in action to the mouse), which is connected to the personal computer and programmed to capture the deviations of the hand movement and measure its latency. The ratio of the horizontal deviations to the vertical deviations is termed as skew ratio. The latency (reaction time) was measured and skew ratio was calculated for each subject. Males and females subjects were examined. A comparative study of the effect of reaction time (latency) on skew ratio of the horizontal hand movement for controls and others suffering from mental illness have been conducted.

Controls mean reaction time was equal to $2.15 \mathrm{~s}$ and $5.28 \mathrm{~s}$ for males and females, respectively; while they were equal to $7.32 \mathrm{~s}$ and $7.33 \mathrm{~s}$ for male and female patients, respectively. Reaction time was lower for controls compared to that of patients; which ascertain its dependence on state of health and gender. Controls mean skew ratio was equal to 36.5 for males and equal to 27.6 for females, while this ratio was equal to 4.9 for abnormal males and was equal to 3.8 for abnormal females respectively. A high value of skew ratio and a small value of reaction time are indicators of sound hand movement and hence good health while low values are indicators of poor hand movement and hence poor health. When gender was considered the skew ratio for normal males to normal females was found to be as high as 1.32:1, while that for abnormal males to females was found to be as high as 1.29: 1 which ascertain its dependence on gender. No relationship was noticed between Skew ratio and Reaction time.

\section{Keywords:}

Computer interface, Hand kinematics, Latency, Hand tremor, Deviation, Skew ratio. 


\section{Introduction:}

Therapists use observation to evaluate the movement deficits of subjects with neurological and muscular diseases. Observation of this kind is used to generate hypothesis regarding impaired muscle activity due to mental disturbances, and in effect to form bases for clinical decision about treatment. Studies of this kind are moderately reliable [1]. A study was conducted by [2] in which the results of an upper-limb positioning task performed by patient were compared to those obtained from control participants. Patients tended to undershoot the target to a greater extent than control participants did, this finding suggests abnormality in the sensory input. [3] Found out that reaction times for patients were longer than that for controls, which is confirmed in this study. In a previous work conducted by [4] hand movement was investigated for adults and was affected by age and gender. It was notices by [5] that the kinematics of pointing was affected by age. Health assessment based on hand movement is considered by [6], which is also supported by the work done by [7]. Hand movement is always accompanied with certain amount of time delay which can be defined as the reaction time of the hand or the subject response to a certain stimulus; this can vary with the speed of the hand movement as reported by [8] and [9]. A computer measurement of latency (reaction time) was reported by [10] who found that it was affected by state of health, age and gender, Motion of the hand is always accompanied with certain amount of skew which can be defined as a type of bias or tilt in movement, this skew can be measured as an angle or an inclination. Path curvature and position of maximum deviation (angle) from straightness were lower for downward than for upward movements, angle measured was also found to vary with the speed of the hand movement, in addition, the position of maximum deviation from straightness became progressively higher with increase in duration for both upward and downward movements and is a function of speeds. This study however builds on a previous work by taking into consideration other parameters such as the skew ratio and its dependence on latency in an attempt to give a quantitative measure of the effect of state of health age and gender on hand movement, and to check if such parameters can be used as a diagnostic tool for human health. It is known that a large reaction time is that caused by mental retardation due to poor attention and poor hand brain coordination and hence mental retardation [11]. This research however, postulates that the above mention parameters reaction time and skew are affected by state of health, gender and age of the subject conducting the test and also inspect the relationship between them. If this assumption is made valid then the above parameters can be considered as an important tool for the assessment of the state of health based on hand kinematics, and for diagnosing certain diseases such as mental illness and hand tremor especially for people suffering from multiple scleroses. As far as the collection of data was concerned the use 
of electrical transducers was avoided due to the presence of noise [12]. Cinematography is also avoided due to the time latency induced between shots, which are known to reduce the accuracy of the test. In contrast a light pen and the personal computer are used to capture the signal and they suffice for this type of work. The light pen output depends totally on the agent moving it, which is the hand in this case; Visual Basic 6 is used as the programming language [13].

\section{Method:}

\subsection{Participants:}

Four participant groups took part in this research. The first group comprised 50_Male controls aged 14 to 67 years. The second group comprised 27 Female controls aged 5 to 49 years. None of the participants reported any skeletomuscular hand or neurological problems [14]. The third group comprised 58 Male patients aged 10 to 55 years. The fourth group comprised 43 Female patients aged 6 to 42 years. Participants in all groups were required to have normal vision. Controls were required to be able to move the hand freely in both flexion and extension.

\subsection{Equipments:}

A personal computer, a Table $72 \mathrm{~cm}$ high and a light pen are used for conducting the test. The light pen is a computer interface devices similar in action to the mouse but the difference is that the light pen is held between the tips of the thump at one end and the fourth finger at the other end provides a measure of displacement in response to the lateral hand movement, and is connected to the personal computer through the USB port.

The table provides the horizontal datum for the hand to rests while performing the test and keeps the hand at constant height which eliminates extra parameters that might degrade the results of the experiment. The set up used by [15] for the measurements of hand latency is used again in this study, with a slight modification made to the software prepared, to count for the calculations of the newly proposed parameters such as the skew ratio.

\subsection{Procedure:}

Participants were shown how to hold the light pen while seated comfortably in front of a personal computer screen with the hand resting at the surface of the flat table and asked to move the hand holding the light pen horizontally many time to get accustomed to the test, in an effort to reduce measurement errors [16]. They were also instructed to keep 
their hand steady while moving it and move it carefully to produce a straight horizontal trajectory.

A stimulus is used to start the test, which is a line segment that appears on the screen in front of the participant for a short period, and then disappears, which signals the start of the test.

The participant is asked to responds to the stimulus by moving the hand holding the light pen horizontally to match that of the line segment just disappeared, in effect a line is traced on the screen which resembles the hand movement, the test ends when the participant releases the light pen. The direction of movement was from right to left.

The hand movement, which is a reflection of the light pen motion, produces a positional signal, which in turn is transformed into a digital signal suitable for the computer to work on.

\subsection{Analysis:}

Reaction time is the period elapsed from the disappearance of the sample line segment off the computer screen Tsoff to the moment of the onset of hand movement i.e. start of trace Tton (on the computer screen) as shown in equation 1 :

Reaction Time $(\mathrm{ms})=$ Tton - Tsoff

Coordinates of the position of the light pen are recorded by the computer as the true position of the hand in the form of an array of $\mathrm{x}$ and $\mathrm{y}$ values for further analysis.

In all cases and for all subjects the hand movement had an either positive or negative tilt (skew); consequently this skew of the hand is recorded as well as the lateral movement. Skew ratio as defined by equation 2 below is measured as a function of reaction time (latency), state of health, age and gender of the subject.

Assuming the starting coordinates of the hand are $x(i)$ and $y(i)$ where $x$ represents the horizontal coordinate and y represents the vertical coordinate, the index (i) represents a point in the line through which the hand moves and at the end includes all points representing the full motion, therefore; the net horizontal to vertical displacement is given by equation 2 :

Skew Ratio $=\Sigma x(i) / \Sigma y(i)$

$\Sigma x(i)$ and $\Sigma y(i)$ are horizontal and vertical tilt

For $\mathrm{i}=1,2,3$ to $\mathrm{n}$. where $\mathrm{n}$ is an integer represents the last point in the hand trajectory. Skew ratios and latency are calculated for every subject using the above formulas in order to check for the validity of any relationship between skew ratio and latency and its possible dependence on state of health, age and gender. 
This figure shows that skew ratio is not dependent on reaction time at all ages.

\section{Results and Discussion:}

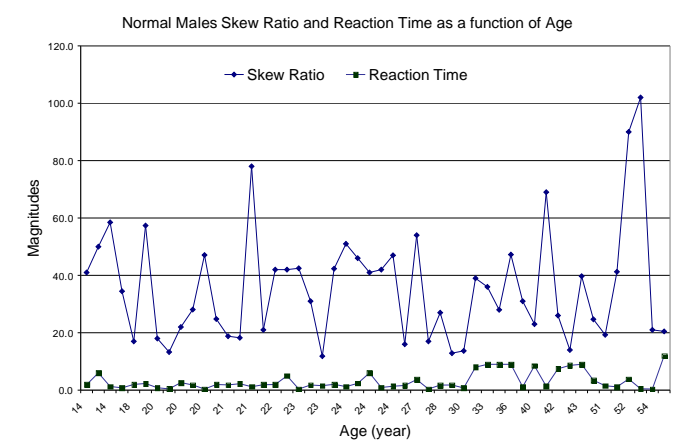

Figure(1): Male controls Reaction Time and Skew Ratio as a function of Age.

Mean Reaction time was equal to $2.15 \mathrm{~s}(\mathrm{SD}=1.96 \mathrm{~s})$. In general reaction time seems to increase with age to different degrees. Correlation factor of reaction time with age was 0.48 which ascertain the dependence of these parameters on age.

Mean Skew Ratio was equal to 36.5 ( $\mathrm{SD}=19.6$ ). Skew Ratio measured fluctuates independent of age, the correlation factor calculated between Skew Ratio and Age for this group was equal to 0.09 , which proves the slight dependence of skew ratio on age.

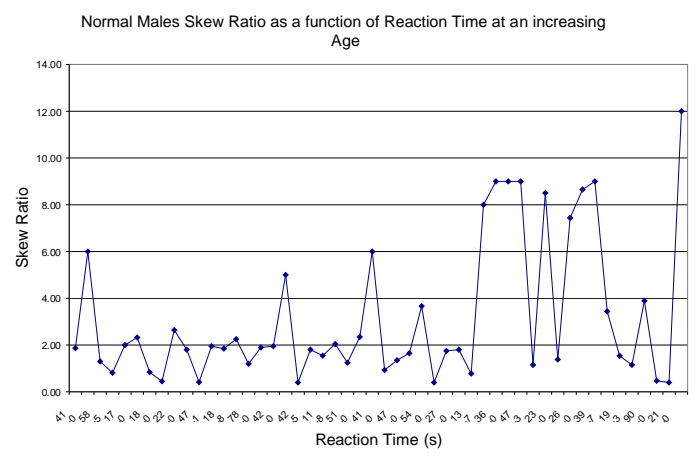

Figure(2): Male controls Skew Ratio as a function of Reaction Time at an increasing Age. 


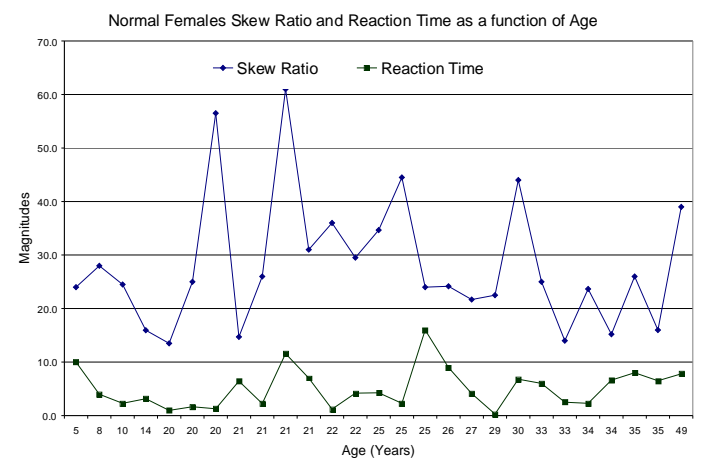

Figure(3): Female Controls Reaction Time and Skew Ratio as a function of Age.

Mean Reaction time was equal to $5.28 \mathrm{~s}(\mathrm{SD}=3.67 \mathrm{~s})$. Both reaction time and Skew Ratio are not affected by age. Correlation factor of reaction time with age was 0.12 . From Figures 1 and 3 shown above it was noticed that the mean values of the reaction time for female controls is more than double that for male controls.

Mean Skew Ratio was equal to 27.6 (SD = 13.2). Skew Ratio measured fluctuates independent of age, the correlation factor calculated between Skew Ratio and Age for this group was equal to 0.008 , which proves the independency between the two parameters.

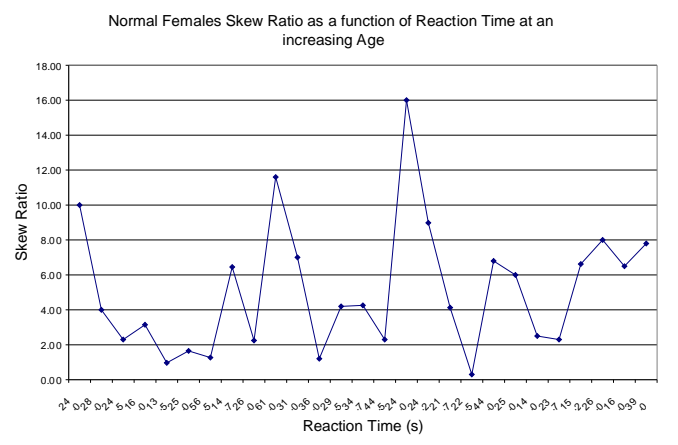

Figure(4): Female controls Skew Ratio as a function of Reaction Time at an increasing Age.

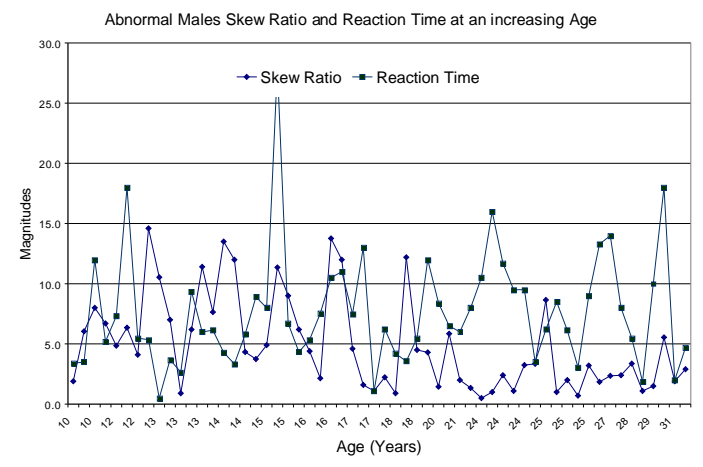

Figure(5): Abnormal Males Skew Ratio and Reaction Time at an increasing Age. 
Mean Reaction time was equal to $7.32 \mathrm{~s}(\mathrm{SD}=4.48 \mathrm{~s})$. The mean reaction time was far higher than that for normal subjects, which indicates that the participant is delaying the action of moving the hand; this is the result of mental retardation. In a previous study conducted by [17] it was reported that quick movement of the hand can lead to deviation in the upward or the downward direction, which are considered as error in the movement, such deviation are also features of disability. Reaction time did not seem to vary with increasing age. Correlation factor of reaction time with age was equal to 0.03. Mean Skew Ratio was equal to 4.9 (SD =2.6). Skew Ratio measured fluctuates but with decreasing value as age increased, the correlation factor calculated between Skew Ratio and Age for this group was equal to -0.36 , which suggests that the skew ratio is reduced with increased age as can be seen from the right hand side of the figure.

This figure shows that skew ratio is independent of reaction time, and especially at increased age.

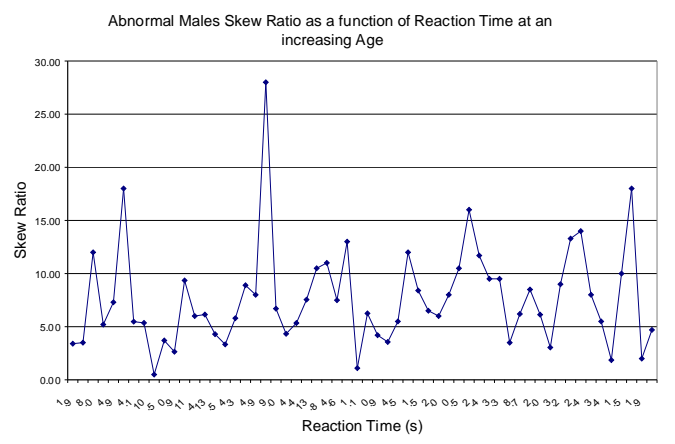

Figure(6): Abnormal Males Skew Ratio as a function of Reaction Time at an increasing Age.

This figure shows that skew ratio is not dependent on reaction time at all ages. Large fluctuation is noticed which is more than that measured for other groups.

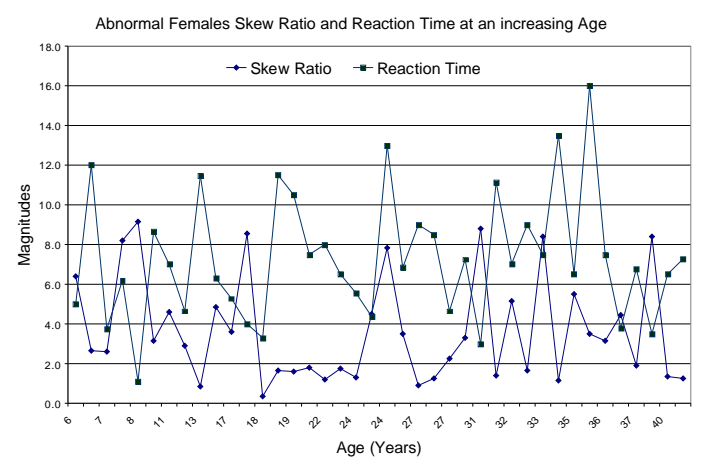


Figure(7): Abnormal Females Skew Ratio and Reaction Time at an increasing Age.

Mean Reaction time was equal to $7.33 \mathrm{~s}(\mathrm{SD}=3.23 \mathrm{~s}$ ) and was the highest of all groups. Hand traces performed were badly affected with vertical deviations this is a sign of mental retardation [18]. Correlation factor of reaction time with age was equal to 0.12 .

Mean Skew Ratio was $3.8(\mathrm{SD}=2.3)$. Skew Ratio measured fluctuates with age of the subjects, the correlation factor calculated between Skew Ratios and Age for this group was equal to -0.13 , which indicates slight reduction of skew ratio with increased age.

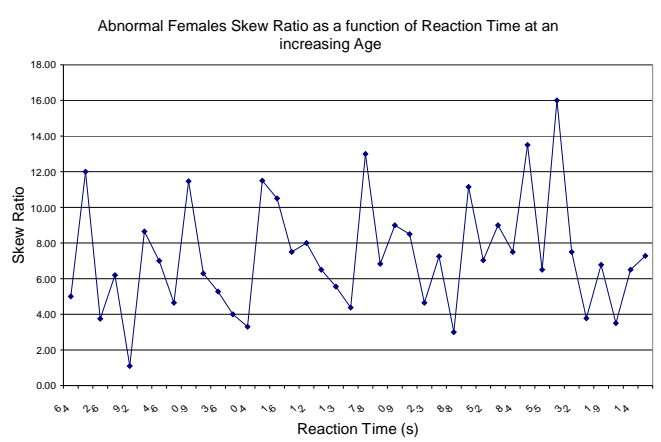

Figure(8): Abnormal Females Skew Ratio as a function of Reaction Time at an increasing Age.

This figure shows that skew ratio is not dependent on reaction time at all ages. Large fluctuation is noticed which is more than that measured for control group.

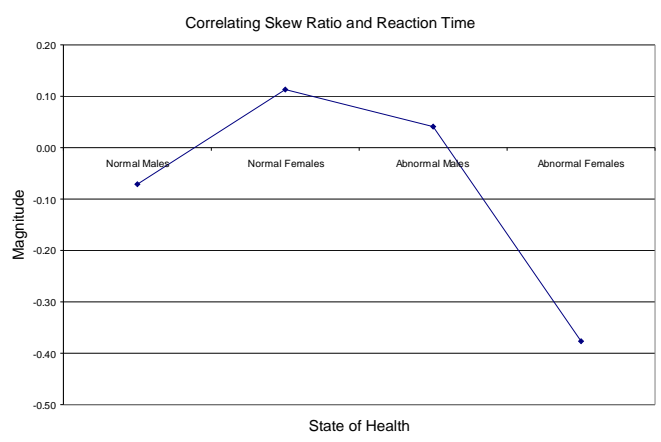

Figure(9): Correlating Skew Ratio and Reaction Time as a function of state of health and gender.

Small values of correlation factor are noticed for all groups which prove that the two parameters are independent of each other. 


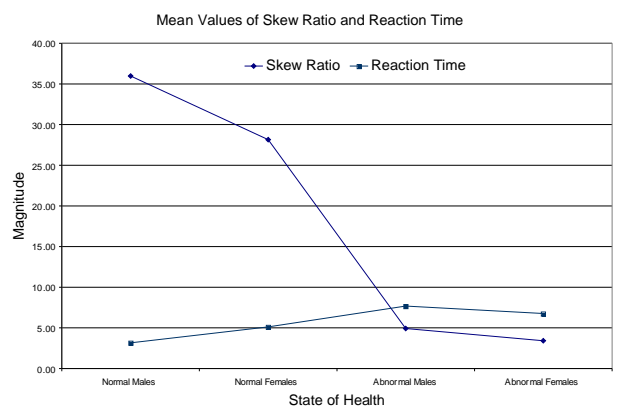

Figure(10): Mean Values of Skew Ratio and Reaction Time.

This figure is of great importance since it clearly emphasizes six main points:

1- Skew Ratio is high for normal subjects and low for abnormal subjects.

2- Normal males average skew ratios are approximately 7.45 times those calculated for abnormal males, and normal females average values are approximately 7.26 times those for abnormal females

3- Points 1 and 2 above justify the role of skew ratio as a diagnostic tool for the assessment of the state of health.

4- Skew Ratio is dependent on gender, since normal males average values are approximately 1.32 times that of normal female subjects, and abnormal males average values are approximately 1.29 times that of abnormal female subjects.

5- Controls have the lowest reaction time, followed by patients; this is of diagnostic value too.

6- Skew ratio is independent of reaction time.

These parameters (skew ratio and reaction time) have not been investigated in this sense before; it might be appropriate to refer to the closest possible related studies such as the work done on latency of hand movement. [19] considered latency as a function of age and state of health and reported its dependency on both of them, it can be said that the results are consistent with the results of that work. Results are also consistent with those reported by [20] where the central nervous system was found to plan the movement of the hand. The model therefore is dependent on a combination of sensory and predictive processes, and measurements based on those may be impaired by poor state of health, gender and old age. Mentally retarded subjects and elderly subjects maintained low values of ratios, which

are the result of poor hand mind coordination and other factors mentioned in the study. [21] considered vertical deviation as a function of age for normal subjects and reported dependency of deviation on age, it can be said that the results are to a small degree consistent with that work but the difference is due to the nature of the parameters used, since in that study a one dimensional analysis was used, while in this study two dimensional approach was adapted which fully represents the natural hand movement. Results are more consistent with those reported by [22] where the central nervous 
system was found to operate an internal model in planning movement of hand. With an intended hand movement the model predicts the associated deviation, such deviation depends on sensory updating about the current state of mind [23].

The same effect is noticed with the skew ratio. It can be said that the results are consistent with the results of others work. Mentally retarded subjects and elderly subjects maintained high values which are the result of poor hand mind coordination.

\section{Conclusions:}

Skew ratio and reaction time were independent of each other.

Mental retardation worsens the two parameters of the hand movement.

Skew ratio as a function of reaction time is not affected by age or gender.

System and Software prepared for this work were sufficient for the intended study.

\section{References:}

1. Goodkin, R. Diller L, "Reliability among physical therapists in diagnosis and treatment of gait deviations in hemiplegics", Percept Mot Skills, Vol. 37, pp. 727734, 1973.

2. Dichgans et al, "A defect of kinesthesia in Parkinson's disease. Movement Disorders", Vol. 10, no. 4, pp. 460-465, 1995.

3. Lewis, G. N., Byblow, \& Walt, "Stride length regulation in Parkinson's disease, The use of extrinsic, visual cues", Brain, Vol. 123, no. 10, pp. 2077-2090, 2000.

4. DeGoede et al, "How quickly can healthy adults move their hands to intercept an approaching object? Age and gender effects", The Journas of gerontology, Vol. 56A, Iss 9, pp. 584-589, 2001.

5. Behav. J, "Information processing and movement optimization during development: kinematics of cyclical pointing in 5- to 11-year-old children", Phys Ther, Vol. 135, no. 2, pp. 183-95. June 2003.

6. Moseley et al, "Interrater Reliability of the TEMPA for the Measurement of Upper Limb Function in Adults With Traumatic Brain Injury", The Journal of Head Trauma Rehabilitation, Vol. 18, No. 6, pp. 526-531, 2003.

7. Isabel et al, "Anatomic Distribution of Sensory Symptoms in the Head and Their Relation to Neck Pain, Psychosocial Variables, and Occupational Activities", American Journal of Epidemiology, Vol. 157, no. 6, pp. 524-530, 2003.

8. Schieppati, M, "Trajectories of arm pointing movements on the sagittal plane vary with both direction and speed", Exp Brain Res, Vol. 148, no. 4, pp. 498-503, Feb 2003. 
9. Eric F, "Event Identification in Movement Recordings by Means of Qualitative. Neuroinformatics; Phys Ther, Vol. 1, pp. 239-258, 2003.

10. Muheilan M, "Measurements of Eye Latency as a Function of Stimulus Frequency", Dirasat, Engineering Sciences, Vol. 25, no.1, pp. 129-135, 1998.

11.Jackson, A, " Automated Electrooculography a microprocessor Application example", Journal of Medical Engineering and Technology, Vol. 4, no. 6, pp. 34-45, 1980.

12. Muheilan M, "Using Personal Computer to Measure Vertical Deviation From A Prescribed Horizontal Track", Mansoura Engineering Journal, Vol. 28, no. 3, pp. 2246, 2003.

13.Deitel, h., and Deitel, P, Visual basic 6, Prentice hall, New Jersey, pp. 11-41. 1999.

14.Martin and Douglas, "Statistics Notes: Measurement error", BMJ, Vol. 313, pp. 740744, 1996.

15. Muheilan M, "Computer Measurements of Latency and the Angle of Deviation of the Hand for Normal Controls and Subjects Suffering from Mental Illness", Abhath Al-Yarmouk Pure Science and Engineering Series , Vol.13, No.2, pp.335-354, 2004.

16. Martin and Douglas, "Statistics Notes: Measurement error", BMJ, Vol. 313, pp. 740744, 1996.

17. Muheilan M, "Computer assessment of Hand deviation A comparative study", ICCTA 2006,5-7 September 2006, Alexanderia, Egypt.

18.dysfunction: Clinical and kinematic analysis", Brain, Vol. 123, no.8, pp. 1568-1580, Aug 2000.

19. Muheilan M, "Computer Measurements of Latency and the Angle of Deviation of the Hand for Normal Controls and Subjects Suffering from Mental Illness", Abhath Al-Yarmouk Pure Science and Engineering Series , Vol.13, No.2, pp.335-354,2004.

20.Gilles M, et al, "Age-Related changes in Grip Force and Dynamics of Hand Movement", Journal of Motor Behavior, Vol. 35, no. 1, pp.79-85, 2003.

21. Muheilan M, "Using Personal Computer to Measure Vertical Deviation From A Prescribed Horizontal Track", Mansoura Engineering Journal, Vol. 28, no. 3, pp. 2246, 2003.

22. Gilles M, et al, "Age-Related changes in Grip Force and Dynamics of Hand Movement", Journal of Motor Behavior, Vol.35, no.1, pp.79-85, 2003.

23.G. lewis, et al, "Effector-Specific Visual Information Influences Kinesthesis and Reaction Time Performance in Parkinsons Disease", Journal of motor behaviour, Vol.35, no.2, pp. 99-107, 2003. 